\title{
Assessment of GPS velocity accuracy for the Basin and Range Geodetic Network (BARGEN)
}

\author{
J. L. Davis and R. A. Bennett \\ Harvard-Smithsonian Center for Astrophysics, Cambridge, Massachusetts, USA
}

B. P. Wernicke

California Institute of Technology, Pasadena, California, USA

Received 21 January 2003; revised 11 March 2003; accepted 13 March 2003; published 11 April 2003.

[1] We assess the accuracy of horizontal velocity estimates from the Basin and Range Geodetic Network (BARGEN), a continuous GPS network that has been in operation since 1996. To make this quantitative assessment, we use a procedure that we term the "whole-error" method. In this method, the measure of the velocity errors is the root-mean-square (RMS) residual velocity relative to a simple geophysical model. This method produces a conservative estimate of the uncertainties, since errors in the geophysical models also contribute to the RMS residual. Using estimates from two different BARGEN subnetworks, the Northern Basin and Range and the Yucca Mountain Cluster, we determine velocity uncertainties of

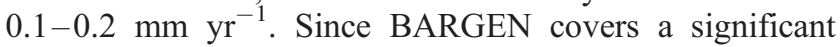
fraction of area of the proposed Plate Boundary Observatory component of EarthScope, our results indicate a good ability of this project to determine highly accurate long-term horizontal crustal velocities and deformation rates in this region. INDEX TERMS: 1208 Geodesy and Gravity: Crustal movements - intraplate (8110); 1243 Geodesy and Gravity: Space geodetic surveys; 1294 Geodesy and Gravity: Instruments and techniques. Citation: Davis, J. L., R. A. Bennett, and B. P. Wernicke, Assessment of GPS velocity accuracy for the Basin and Range Geodetic Network (BARGEN), Geophys. Res. Lett., 30(7), 1411, doi:10.1029/2003GL016961, 2003.

\section{Introduction}

[2] With the advent of continuously operating Global Positioning System stations, it has become possible to determine three-dimensional site velocities using a long time series of site position estimates, sampled daily. With this approach the accuracy of velocity for a series of measurements of timespan $T$ could theoretically decrease as $T^{-3 / 2}$, with the total timespan contributing a factor of $T^{-1}$ and the number of daily position determinations contributing a factor of $T^{-1 / 2}$. It is generally accepted that this theoretical limit, which depends on the daily estimates being uncorrelated, cannot be reached in practice. It is not generally accepted that any particular error model for GPS velocities is most realistic, nor is it understood in detail which factors ultimately limit the accuracy of velocity determinations from GPS.

[3] Although studies aimed at assessment of the accuracy of GPS determinations of position were common a decade

Copyright 2003 by the American Geophysical Union. 0094-8276/03/2003GL016961 ago (see, e.g., Dixon [1991] and references therein), such studies have not kept pace with the advances in positioning ability stemming from a much improved global tracking network, improvements in data processing capabilities and computer hardware, and the use of continuous GPS stations for geophysical studies. In particular, no definitive studies exist that answer the following fundamental questions: (1) Is the estimate of velocity from GPS fundamentally limited by one or more error sources and (2) if so, what are these error sources?

[4] The present trend seems to be to utilize conservative velocity error models [e.g., Dixon et al., 2000] based on power-law or other mathematical models for time-dependent noise [e.g., Agnew, 1992]. Langbein and Johnson [1997] demonstrated that such errors could increase the errors of the velocity determinations relative to the "white noise" value significantly. The source of the noise for the Langbein and Johnson [1997] study was unmodeled motion of the geodetic monument on which a trilateration reflector was mounted. This source of noise has been assumed to predominate, without demonstration, in GPS studies as well [e.g., Zhang et al., 1997]. This approach produces velocity error estimates that are perhaps overly conservative for many sites. Given the resources and efforts invested with existing continuous GPS networks, and the prospects of $\sim 1000$ new sites of the Plate Boundary Observatory (PBO), a revisiting of this important unsolved issue is warranted.

[5] This study represents one step in the process, accurate assessment of the velocity errors for a specific network of continuous GPS sites. This network, the Basin and Range Geodetic Network (BARGEN), covers a significant area of the proposed PBO within the conterminous U.S., and therefore represents the potential ability of this project to determine long-term velocities. Our approach involves using simple yet realistic models for the geographic variability of the measured horizontal velocities. The unweighted root-mean-square (RMS) velocity residual to the best-fit model is a measure of the accuracy of the velocity. (The RMS is calculated in the usual way taking into account the degrees-of-freedom.) This approach lumps errors in the velocity estimates together with errors in the model for the geographic variability; for that reason we call this the "whole-error" method.

[6] Because the whole-error method assesses misfit of the geographic variability of velocity, it is insensitive to common-mode errors. The most likely cause of a commonmode velocity error for networks of the extent of BARGEN is an error in the realization of the velocity reference-frame. 


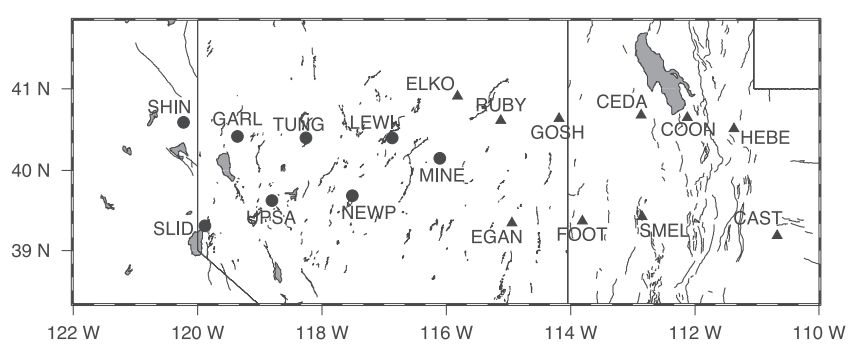

Figure 1. Map of the Northern Basin and Range (NBAR) subnetwork. Triangles indicate the sites used in the analysis.

We thus distinguish between reference-frame errors and the statistical variability inherent in the velocity estimates, and it is the latter that we address.

\section{Analysis}

[7] The BARGEN network consists of 50 continuously operating, deeply anchored [Langbein et al., 1995] GPS stations in the northern and central Basin and Range. For this study, we include data through July 2002. The BARGEN network and the data analysis procedures we use are described in detail by Bennett et al. [2002]. Our velocity determinations result from a three-stage solution employing the GAMIT [King and Bock, 1999] and GLOBK [Herring, 2002] software packages. The "formal" velocity uncertainties are based on the least-squares propagation of scaled observation errors and are intended to represent the statistical uncertainty due to errors in the space geodetic measurements.

[8] The BARGEN network was constructed in a number of phases. In the north of the network, 18 sites in two eastwest lines straddle latitude $40^{\circ} \mathrm{N}$. Eight of these sites began operation between July and September 1996. A second group of four sites began operation in March 1997, while the remaining six sites began operation between July and October 1997. The length of the time series for these sites in our analysis therefore varies between 4.8 and 6.0 years.

[9] In the south of the network, the remaining 32 BARGEN sites are generally centered around Yucca Mountain, although a few are several hundred kilometers distant. For our analysis, we will focus on the main cluster of 17 sites within $100 \mathrm{~km}$ of each other. All of these sites but one began operation in March 1999; the remaining site began operation in the first week of April 1999. Therefore, the data from these sites span slightly more than three years.

[10] The horizontal velocities from each of these two subnetworks form a fairly uniform data set to which we can independently apply the method of whole-error analysis. In the following subsections, we describe these analyses.

\section{The Northern Basin and Range (NBAR) Subnetwork}

[11] The 18 sites of the NBAR subnetwork of BARGEN are shown in Figure 1. The velocity field in this region from both continuous and campaign GPS has been modeled by Bennett et al. [2003] as an east-west series of discrete elastically-deforming microplates. In the east, sites on the Colorado Plateau $(\mathrm{CP})$ province provided a fixed and undeforming reference frame. Moving successively westward, the Eastern Great Basin (EGB) microplate was found to be undergoing east-west extension, whereas the Central Great Basin (CGB) microplate moved westward at a rate of $2.8 \pm 0.2 \mathrm{~mm} \mathrm{yr}^{-1}$ The deformation appears more complicated in the Western Great Basin (WGB), a region that includes the Central Nevada Seismic Belt (CNSB). Using this heterogeneous data set, Bennett et al. [2003] obtained typical root-mean-square (RMS) velocity residuals, relative to the microplate model, of slightly better than $\sim 1 \mathrm{~mm} \mathrm{yr}^{-1}$.

[12] The Bennett et al. [2003] solution provides us with a simple and useful model for one particular subset of the velocity solution. Throughout the northeastern part of the network (those sites on the CP, EGB, and CGB) the Bennett et al. [2003] solution predicts that the northern component of the horizontal velocity is constant. (This solution used the Thatcher et al. [1999] data set as well.) The estimated velocities, with 2- $\sigma$ error bars, are shown in Figure 2. This figure also shows an expanded figure for the north components of velocity (top right), which in fact are fairly constant over this part of the network. We will use these ten values for our analysis. (The sites used are shown as triangles in Figure 1. The correspondence to geodetic region is given in Bennett et al. [2003].)

[13] We calculate that the RMS residual of these velocitycomponent values about a mean value is $0.23 \mathrm{~mm} \mathrm{yr}^{-1}$, with a maximum residual of $0.33 \mathrm{~mm} \mathrm{yr}^{-1}$. The question is, how representative are these 10 sites? It might be, for
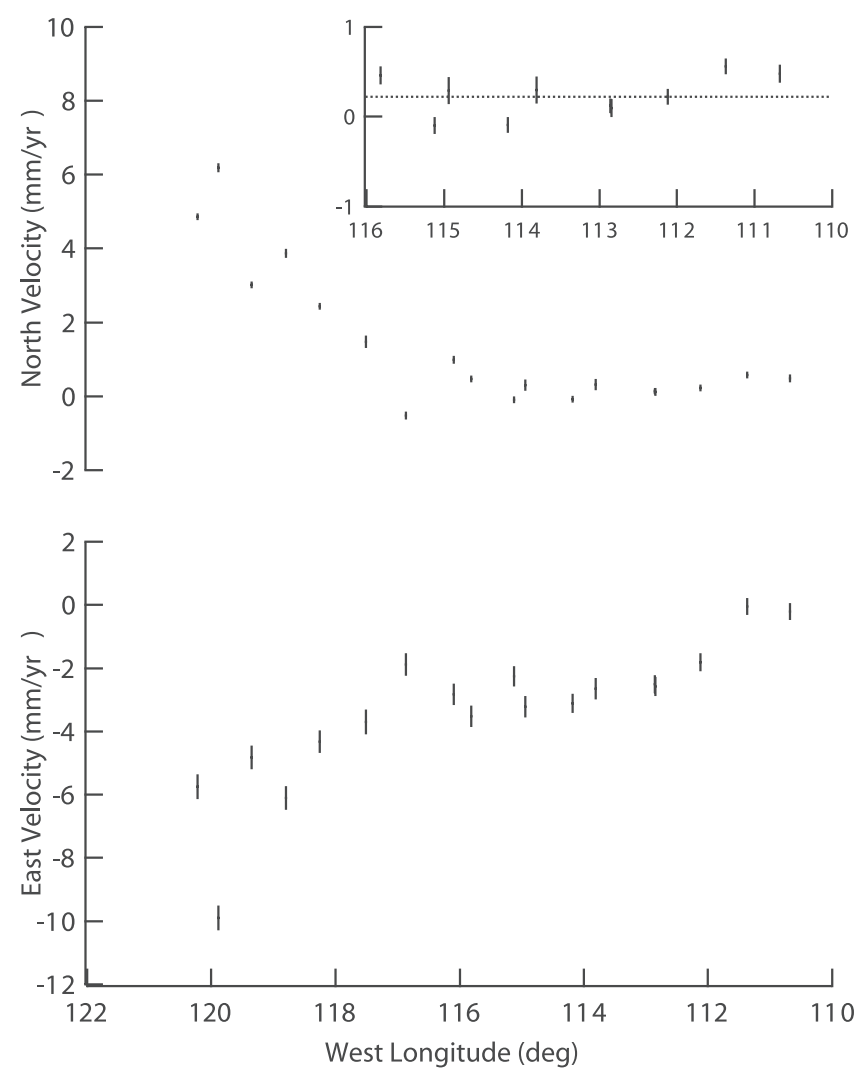

Figure 2. Velocity estimates for NBAR. Error bars are $2-\sigma$. The dotted line in the expanded figure for the north components of velocity (top right) indicates the mean value for these estimates. 
instance, that the quality of these eastern sites is somehow better than the others. This part of the BARGEN network was installed east to west in two phases, with the northern line being installed first. There is nothing known to us, however, to suggest that the monumentation or any other aspect of these sites is better than for any other subset of BARGEN sites. Some of the sites in the western subregion use a different communication scheme that required a longer time for debugging. Thus, it may be that our assumption regarding network homogeneity for the velocity standard deviation is incorrect. It would be difficult to test this hypothesis, but there is no evidence that points to such a heterogeneity.

[14] Could the act of limiting the data set significantly increase the likelihood of obtaining a low RMS value? In other words, given 18 site estimates of north velocity components that actually have a larger standard deviation (say $0.5 \mathrm{~mm} \mathrm{yr}^{-1}$ ), what is the likelihood that 10 of the components have a standard deviation of only $0.2 \mathrm{~mm} \mathrm{yr}^{-1}$. There is, in fact, some selection involved but there is, of course, a geophysical basis for this subdivision. Nevertheless, to investigate the possibility that our result is a statistical fortuity, we performed a Monte Carlo analysis. We repeatedly generated a sequence of 18 independent random numbers (representing the north components of velocity for the 18 sites). Each of the numbers in the sequence was drawn at random from a Gaussian distribution with a mean of zero and a standard deviation of 0.5 (i.e., 0.5

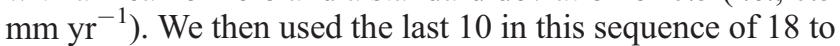
calculate a standard deviation. The likelihood of achieving an RMS value less than or equal to $0.2 \mathrm{~mm} \mathrm{yr}^{-1}$ is only $\sim 0.1 \%$. We conclude, therefore, that it is highly unlikely that our result stems from a statistical selection effect.

[15] Nevertheless, we cannot rule out that a velocity estimate from a particular site does not especially suffer from some error. Site LEWI (Figure 1), for example, has an apparently anomalous southward velocity (Figure 2). This anomaly may be indicative of an error that is especially significant for that site. On the other hand, Wernicke et al. [2000] argue that the velocity for this site, located just east of the CNSB, may be associated with a eastward propagating strain pulse generated by the Pleasant Valley (1915) and Dixie Valley (1954) earthquakes. Thus, this result is not prima facie evidence for a site-dependent error.

\section{The Yucca Mountain Cluster (YMC)}

[16] A map of the YMC sites is shown in Figure 3. Although in the recent past this region has exhibited localized strain anomalies, perhaps in response to the Little Skull Mountain earthquake of 1992 [Wernicke et al., 1998; Savage et al., 2001], the velocity in the region since the April 1999 start of our survey can be described by a constant strain-rate matrix. The predominant strain-rate can be inferred visually from the observed velocities relative to North America, shown with black arrows with 95\% confidence ellipses in Figure 3. A decrease in the northerly component of velocity with eastward direction (about $1 \mathrm{~mm}$ $\mathrm{yr}^{-1}$ over the width of the YMC) can be observed. A fit using a constant strain rates model to describe the average deformation throughout the cluster yields estimates for the principal strain-rate components of $7 \pm 1$ nstrain $\mathrm{yr}^{-1}$ at an

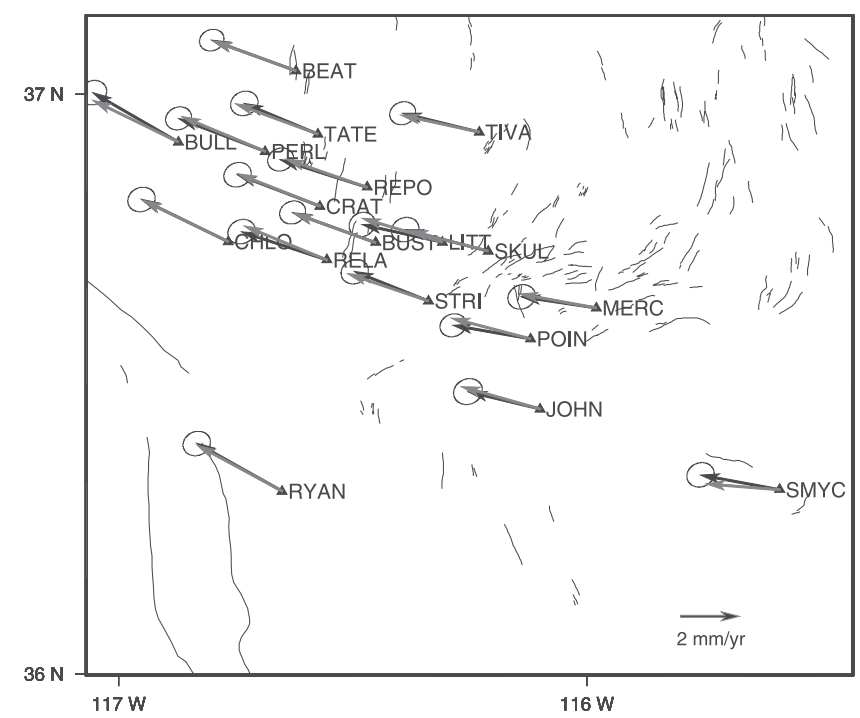

Figure 3. Sites of the Yucca Mountain Cluster (YMC). The estimated velocities and their 95\% confidence ellipses (based on 2- $\sigma$ errors) are shown in black. Values calculated using the simple strain-rate model are shown in gray.

azimuth of $\mathrm{N} 64^{\circ} \pm 2^{\circ} \mathrm{W}$ and $-10 \pm 1$ nstrain $\mathrm{yr}^{-1}$ at $\mathrm{N} 26^{\circ} \pm$ $2^{\circ} \mathrm{E}$ (extension positive). These strain-rate values are smaller than, but in a direction consistent to, the values of $23 \pm 9$ nstrain $\mathrm{yr}^{-1}$ at $\mathrm{N} 78^{\circ} \pm 14^{\circ} \mathrm{W}$ and $-9 \pm 12$ nstrain $\mathrm{yr}^{-1}$ at $\mathrm{N} 13^{\circ} \pm 14^{\circ} \mathrm{E}$ reported for GPS data acquired over about the same aperture for 1993-1998 [Savage et al., 2001]. These latter values are in turn much smaller for a combined GPS (1991-1997) and trilateration (1983-1997) study that focussed on a much smaller aperture near Yucca Mountain, and found that the line equivalent to REPO-LITT was increasing at 50-64 \pm 14 nstrain $\mathrm{yr}^{-1}$ [Wernicke et al., 1998].

[17] The best-fit constant strain-rate model values for the velocities are shown in gray in Figure 3. The RMS difference between the observed and best-fit model values is 0.15 $\mathrm{mm} \mathrm{yr}^{-1}$. (The 18 sites yield 36 velocity components. The model includes a constant value for each component plus four values for the strain-rate matrix for a total of six parameters.) The largest residual is only $0.3 \mathrm{~mm} \mathrm{yr}^{-1}$, for site SMYC, but the number of degrees of freedom for this solution is sufficiently large that omission of this site improves the RMS residual only slightly.

\section{Discussion}

[18] The velocity uncertainties we estimate, $0.23 \mathrm{~mm} \mathrm{yr}^{-1}$ using the north components of NBAR and $0.15 \mathrm{~mm} \mathrm{yr}^{-1}$ for YMC, are perhaps among the best ever reported. This aspect of our results immediately raises the question of whether these accuracy estimates are unrealistically small. We argue that, on the contrary, these accuracy estimates are realistic, for the following reasons: the models we used for the whole-error analysis are geophysically reasonable, we obtain model values consistent with those of prior studies, and data from two separate regions yielded similar results.

[19] Time series of position estimates for all BARGEN sites exhibit obvious temporal structure, implying the exis- 
tence of temporal correlations among errors for the position estimates. Such correlations may result in underestimates of the velocity uncertainties determined using a white-noise only model. For the north components of NBAR, these "formal" white-noise uncertainties range from 0.08 to 0.15 $\mathrm{mm} \mathrm{yr}^{-1}$, for an average of $0.10 \mathrm{~mm} \mathrm{yr}^{-1}$, indicating that the true uncertainties are dominated by the source or sources of error that introduce the temporal correlations. For YMC, these shorter time series yield "formal" white-noise uncertainties of $0.15-0.21 \mathrm{~mm} \mathrm{yr}^{-1}$, for an average of $0.18 \mathrm{~mm}$ $\mathrm{yr}^{-1}$. (The uncertainties for the east velocities are on average $\sim 12 \%$ larger than those for the north.) Thus, for YMC the whole-error estimated uncertainties are slightly smaller than the average white-noise uncertainties.

[20] It is interesting, in fact, that the YMC results are better than the NBAR results, since the timespan for the former is only slightly more than half as long as the average timespan for the latter. We have observed that the temporal structure is more highly spatially correlated for sites of the spatially smaller YMC network. If the position errors are also spatially correlated, then they might induce "commonmode" velocity errors over the YMC and not be reflected in this application of the whole-error method. Such a commonmode signal is not necessarily geodetic error, however, and may fall into the category of modeling error. (Large-scale deformations [e.g., Blewitt et al., 2001] and regional loading signals [e.g., Elósegui et al., 2003] may fall into this category of signal.) Sources of error that could account for spatial correlation include errors in the orbital position of the GPS satellites and errors in the atmospheric propagation delay model. We are currently investigating the spectral content of all the BARGEN time series.

[21] Site-dependent monument wander for these deeplyanchored sites would be detected in our analysis. We can confidently place maximum limits of $0.23{\mathrm{~mm} \mathrm{yr}^{-1}}^{-1}$ (NBAR) and $0.15 \mathrm{~mm} \mathrm{yr}^{-1}$ (YMC) for the contribution of this effect to our velocity uncertainties. Assuming a random-walk model for this effect, the required variance rates are $0.5-0.6 \mathrm{~mm}^{2} \mathrm{yr}^{-1}$ for NBAR (accounting for the range of data timespans) and $0.1 \mathrm{~mm}^{2} \mathrm{yr}^{-1}$ for YMC.

[22] The climate may also play a role in the better results for YMC. Precipitation, both in the winter and summer months, is greater within the NBAR area than within the YMC area. Especially in the late summer, storms in the NBAR area travel in discrete systems across the network. Thus, there is the potential that atmospheric-delay model errors are not only less correlated within NBAR (due to its greater) but are simply larger.

[23] Our velocity-uncertainty results are averages, and apply only to the networks we have analyzed. Errors in velocity determinations may depend on site geology, climate and weather, electromagnetic environment, distance to other sites, satellite orbit model, and data processing choices. Uncertainties inferred with the whole error method are also dependent on the ability to model estimated site velocities. Within these limitations, we have demonstrated an ability to describe subsets of the BARGEN data with simple models, and from these analyses we infer velocity uncertainties of $0.1-0.2 \mathrm{~mm} \mathrm{yr}^{-1}$. Since BARGEN covers a significant fraction of area of the proposed PBO, our results indicate good prospects for this project to determine highly accurate long-term horizontal crustal velocities and deformation rates in this region.

[24] Acknowledgments. This research was supported by the National Science Foundation, the Department of Energy, NASA, the UNAVCO Facility, the California Institute of Technology, the Smithsonian Institution, and the University of Nevada, Reno. We thank P. Elósegui, H. Johnson, and an anonymous reviewer for useful comments on the manuscript. We also thank J. Langbein and R. King for comments on an earlier version of this work.

\section{References}

Agnew, D. C., The time-domain behavior of power-law noises, Geophys. Res. Lett., 19, 333-336, 1992.

Bennett, R. A., J. L. Davis, B. P. Wernicke, and J. E. Normandeau, Space geodetic measurements of plate boundary deformation in the western U.S. Cordillera, in Plate Boundary Zones, edited by S. A. Stein and J. T. Freymueller, AGU Geodyn. Ser., 30, 27-55, 2002.

Bennett, R. A., B. P. Wernicke, N. A. Niemi, A. M. Friedrich, and J. L. Davis, Contemporary strain rates in the northern Basin and Range province from GPS data, Tectonics, doi:10.1029/2001TC001355, 22(2), 1008, 2003.

Blewitt, G., D. Lavallée, P. Clarke, and K. Nurutdinov, A new global mode of Earth deformation: Seasonal cycle detected, Science, 294, 2342-2345, 2001.

Dixon, T. H., An introduction to the Global Positioning System and some geological applications, Rev. Geophys., 29, 249-276, 1991.

Dixon, T. H., M. Miller, F. Farina, H. Z. Wang, and D. Johnson, Presentday motion of the Sierra Nevada block and some tectonic implications for the Basin and Range province, North American cordillera, Tectonics, $19,1-24,2002$.

Elósegui, P., J. L. Davis, J. X. Mitrovica, R. A. Bennett, and B. P. Wernicke, Crustal loading near Great Salt Lake, Utah, Geophys. Res. Lett., 30(3), 1111, doi:10.1029/2002GL016579, 2003.

Herring, T. A., GLOBK: Global Kalman filter VLBI and GPS analysis program, Version 10, Technical Report, Dept. Earth, Atmos., and Planet. Sci., Massachusetts Institute of Technology, Cambridge, Mass., 2002.

King, R. W., and Y. Bock, Documentation for the MIT GPS analysis software: GAMIT, Technical report, Dept. Earth, Atmos., and Planet. Sci., Massachusetts Institute of Technology, Cambridge, Mass., 1999.

Langbein, J., and H. Johnson, Correlated errors in geodetic time series: Implications for time-dependent deformation, J. Geophys. Res., 102, 591-603, 1997

Langbein, J., F. Wyatt, H. Johnson, D. Hamann, and P. Zimmer, Improved stability of a deeply anchored geodetic monument for deformation monitoring, Geophys. Res. Lett., 22, 3533-3536, 1995.

Savage, J. C., J. L. Svarc, and W. H. Prescott, Strain accumulation near Yucca Mountain, Nevada, 1993-1998, J. Geophys. Res., 106, 16,48316,488, 2001.

Thatcher, W., G. Foulger, B. Julian, J. Svarc, E. Quilty, and G. Bawden, Present day deformation across the Basin and Range province, western United States, Science, 283, 1715-1718, 1999.

Wernicke, B. P., J. L. Davis, R. A. Bennett, P. Elósegui, M. J. Abolins, R. A. Brady, M. A. House, N. A. Niemi, and J. K. Snow, Anomalous tectonic strain accumulation in the Yucca Mountain area, Nevada, Science, 279, 2096-2098, 1998.

Wernicke, B., A. M. Friedrich, N. A. Niemi, R. A. Bennett, and J. L. Davis, Dynamics of plate boundary fault systems from Basin and Range Geodetic Network (BARGEN) and geologic data, GSA Today, 10(11), 1-7, 2000 .

Zhang, J., Y. Bock, H. Johnson, P. Feng, S. Williams, J. Genrich, S. Wdowinski, and J. Behr, Southern California Permanent GPS Geodetic Array: Error analysis of daily position estimates and site velocities, J. Geophys. Res., 102, 18,035-18,055, 1997.

R. A. Bennett and J. L. Davis, Harvard-Smithsonian Center for Astrophysics, 60 Garden Street, MS 42, Cambridge, MA 02138, USA. (rbennett@cfa.harvard.edu; jdavis@cfa.harvard.edu)

B. P. Wernicke, Division of Geological and Planetary Sciences, California Institute of Technology, Pasadena, CA 91125, USA. (brian@gps.caltech. edu) 